\title{
Moments of an exponential functional of random walks and permutations with given descent sets
}

\author{
Tamás Szabados*†and Balázs Székely \\ Budapest University of Technology and Economics
}

Running head: Moments and permutations

\begin{abstract}
The exponential functional of simple, symmetric random walks with negative drift is an infinite polynomial $Y=1+\xi_{1}+\xi_{1} \xi_{2}+\xi_{1} \xi_{2} \xi_{3}+\cdots$ of independent and identically distributed non-negative random variables. It has moments that are rational functions of the variables $\mu_{k}=\mathbf{E}\left(\xi^{k}\right)<1$ with universal coefficients. It turns out that such a coefficient is equal to the number of permutations with descent set defined by the multiindex of the coefficient. A recursion enumerates all numbers of permutations with given descent sets in the form of a Pascal-type triangle.
\end{abstract}

\section{Introduction}

The present work was induced by the following problem from probability theory, cf. [4. Let $\left(X_{j}\right)_{j=1}^{\infty}$ be a sequence of independent and identically distributed random variables with $\mathbf{P}\left(X_{j}= \pm 1\right)=\frac{1}{2}$. Further, let $S_{0}=0, S_{k}=\sum_{j=1}^{k} X_{j}$ $(k \geq 1)$ be a simple, symmetric random walk. Introduce the following exponential functional $Y$ of the random walk with negative drift:

$$
Y=\sum_{k=0}^{\infty} \exp \left(S_{k}-k \nu\right)=1+\xi_{1}+\xi_{1} \xi_{2}+\cdots, \quad \xi_{j}=\exp \left(X_{j}-\nu\right)
$$

where $\nu>0 . Y$ is an approximation of a widely investigated exponential functional of Brownian motion, important for example in studying Asian options of financial mathematics. To generalize it somewhat, let $\left(\xi_{j}\right)_{j=1}^{\infty}$ be independent and identically distributed random variables, $\xi_{j} \geq 0$. Consider first the finite

\footnotetext{
*Corresponding author, address: Department of Mathematics, Budapest University of Technology and Economics, Müegyetem rkp 3, H ép V em, Budapest, 1521, Hungary, e-mail: szabados@math.bme.hu

†Research supported by the French-Hungarian intergovernmental grant "Balaton" F$39 / 2000$.

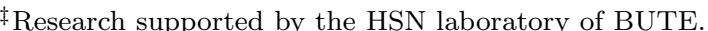

2000 MSC. Primary 05A05, 05A19. Secondary 60C05.

Key words and phrases. Random walk, exponential functional, permutations with given descent sets, Pascal's triangle, infinite polynomials of random variables.
} 
polynomials

$$
\begin{aligned}
Y_{n} & =1+\xi_{1}+\xi_{1} \xi_{2}+\cdots+\xi_{1} \cdots \xi_{n} \\
& =1+\xi_{1}\left(1+\xi_{2}+\xi_{2} \xi_{3}+\cdots+\xi_{2} \cdots \xi_{n}\right) \quad(n \geq 1) .
\end{aligned}
$$

This equation implies the following equality in distribution (denoted by $\stackrel{d}{=}$ ): $Y_{n} \stackrel{\mathrm{d}}{=} 1+\xi Y_{n-1}$, where $\xi \stackrel{\mathrm{d}}{=} \xi_{1}$ and $\xi$ is independent of $Y_{n-1}$. Since $Y_{n} \nearrow Y=$ $1+\xi_{1}+\xi_{1} \xi_{2}+\xi_{1} \xi_{2} \xi_{3}+\cdots$ as $n \rightarrow \infty$, in the limit we get a stochastic difference equation for the infinite polynomial $Y$ :

$$
Y \stackrel{\mathrm{d}}{=} 1+\xi Y,
$$

where $\xi$ is independent of $Y$.

Then using the binomial theorem, the following recursion can be obtained for the $p$ th moment $e_{p}=\mathbf{E}\left(Y^{p}\right)$ of $Y$ for any positive integer $p$ :

$$
e_{p}=\frac{1}{1-\mu_{p}} \sum_{k=0}^{p-1}\left(\begin{array}{l}
p \\
k
\end{array}\right) \mu_{k} e_{k}
$$

supposing $\mu_{p}<1$, where $\mu_{k}=\mathbf{E}\left(\xi^{k}\right), e_{k}=\mathbf{E}\left(Y^{k}\right), k \geq 0$. We mention that $\mathbf{E}\left(Y^{p}\right)<\infty$ if and only if $\mu_{p}<1$, and then $\mu_{k}<1$ for any $1 \leq k<p$ as well, cf. [4] Theorem 2]. Observe that (2) defines a recursive sequence $e_{p}=e_{p}\left(\mu_{1}, \ldots, \mu_{p}\right)$ for $p \geq 1$ with $e_{0}=1$, irrespective of any probability theory background. In this recursion the $\mu_{k}$ 's may be considered as variables that may take any value except 1 for $k \geq 1$. Thus from now on we always suppose that $\mu_{k} \neq 1$ for $k \geq 1$ and $\mu_{0}=1$.

It follows from (2) by induction that $e_{p}$ is a rational function of the variables $\mu_{1}, \ldots, \mu_{p}$ for any integer $p \geq 1$ :

$$
e_{p}=\frac{1}{\left(1-\mu_{1}\right) \cdots\left(1-\mu_{p}\right)} \sum_{\left(j_{1}, \ldots, j_{p-1}\right) \in\{0,1\}^{p-1}} a_{j_{1} \ldots j_{p-1}}^{(p)} \mu_{1}^{j_{1}} \cdots \mu_{p-1}^{j_{p-1}},
$$

where the coefficients of the numerator are universal constants, being independent of the parameters in the recursion.

These coefficients $a_{j_{1} \ldots j_{p-1}}^{(p)}$ make a symmetrical, Pascal's triangle-like table if each row is listed in the increasing order of the binary numbers $j_{p-1} 2^{p-2}+$ $\cdots+j_{1} 2^{0}$, defined by the multiindices $\left(j_{1}, \ldots, j_{p-1}\right)$, see the rows $p=1, \ldots, 5$ in Table 1.

Two natural questions may arise at this point, independently of any probability theory background mentioned above. First, suppose that one defines a recursive sequence $\left(e_{p}\right)_{p=1}^{\infty}$ by (2) with coefficients $a_{j_{1} \ldots j_{p-1}}^{(p)}$ given by (3). Can one attach any direct mathematical meaning to these coefficients $a_{j_{1} \ldots j_{p-1}}^{(p)}$ then? The answer is yes, and rather surprisingly (as was conjectured in [4]), the coefficient $a_{j_{1} \ldots j_{p-1}}^{(p)}$ is equal to the number of permutations $\pi \in S_{p}$ having descent $\pi(i)>\pi(i+1)$ exactly where $j_{i}=1,1 \leq i \leq p-1$, cf. Theorem 1 below.

Second, can one give a direct way to evaluate the coefficients $a_{j_{1} \ldots j_{p-1}}^{(p)}$ ? The affirmative answer to this question is partly included in the previous answer, since several formulae are known for the number of permutations with given descent sets. However, an apparently new recursion was conjectured in 4, which is analogous to the recursion of binomial coefficients in the ordinary Pascal's triangle. The proof of this is the content of Lemma 2 below. 
Table 1: The Pascal's triangle-like table of the coefficients

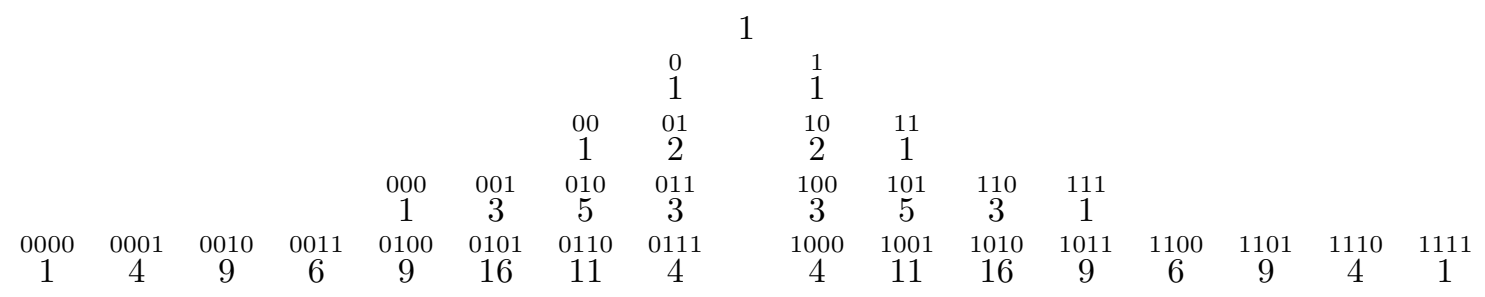

\section{The results}

In the next lemma we establish a direct recursion for the coefficients $a_{j_{1} \ldots j_{p-1}}^{(p)}$.

Lemma 1. Fix a multiindex $\left(j_{1}, \ldots, j_{p-1}\right) \in\{0,1\}^{p-1}$. Let $S$ be the set of indices $k$ where $j_{k}=1$ :

$$
S=\left\{s_{1}, \ldots, s_{m}\right\}=\left\{k: j_{k}=1,1 \leq k \leq p-1\right\}, \quad m=\sum_{k=1}^{p-1} j_{k} .
$$

Then the coefficient $a_{j_{1} \ldots j_{p-1}}^{(p)}$ defined by (3) can be obtained by the recursion

$$
\begin{aligned}
a_{j_{1} \ldots j_{p-1}}^{(p)} & =\sum_{k=0}^{p-1}\left(\begin{array}{l}
p \\
k
\end{array}\right) j_{k}(-1)^{j_{k+1}+\cdots+j_{p-1}} a_{j_{1} \ldots j_{k-1}}^{(k)} \\
& =\sum_{l=0}^{m}\left(\begin{array}{l}
p \\
s_{l}
\end{array}\right)(-1)^{m-l} a_{j_{1} \ldots j_{s_{l}-1}}^{\left(s_{l}\right)}
\end{aligned}
$$

where, by definition, $a^{(0)}=1, j_{0}=1, s_{0}=0$ and -1 powered to an empty sum is 1 .

Proof. The second equality in (5) is a direct consequence of the definitions above. To show the first equality, substitute (3) into (2):

$e_{p}=\frac{1}{1-\mu_{p}} \sum_{k=0}^{p-1}\left(\begin{array}{l}p \\ k\end{array}\right) \frac{\mu_{k}}{\left(1-\mu_{1}\right) \cdots\left(1-\mu_{k}\right)} \sum_{\left(j_{1}, \ldots, j_{k-1}\right) \in\{0,1\}^{k-1}} a_{j_{1} \ldots j_{k-1}}^{(k)} \mu_{1}^{j_{1}} \cdots \mu_{k-1}^{j_{k-1}}$.

Here, multiplying by the common denominator and then collecting the coefficients of $\mu_{1}^{j_{1}} \cdots \mu_{p-1}^{j_{p-1}}$ for each $\left(j_{1}, \ldots, j_{p-1}\right)$ we obtain

$$
\begin{aligned}
e_{p} & \left(1-\mu_{1}\right) \cdots\left(1-\mu_{p}\right) \\
& =\sum_{k=0}^{p-1} \sum_{\left(j_{1}, \ldots, j_{k-1}\right) \in\{0,1\}^{k-1}}\left(\begin{array}{l}
p \\
k
\end{array}\right) a_{j_{1} \ldots j_{k-1}}^{(k)} \mu_{1}^{j_{1}} \cdots \mu_{k-1}^{j_{k-1}} \mu_{k}\left(1-\mu_{k+1}\right) \cdots\left(1-\mu_{p-1}\right) \\
& =\sum_{\left(j_{1}, \ldots, j_{p-1}\right) \in\{0,1\}^{p-1}} \mu_{1}^{j_{1}} \cdots \mu_{p-1}^{j_{p-1}} \sum_{k=0}^{p-1}\left(\begin{array}{l}
p \\
k
\end{array}\right) a_{j_{1} \ldots j_{k-1}}^{(k)} j_{k}(-1)^{j_{k+1}} \cdots(-1)^{j_{p-1}} .
\end{aligned}
$$

This and (3) imply the first equality in (5) . 
Now we turn to the proof of the equality of the coefficient $a_{j_{1} \ldots j_{p-1}}^{(p)}$ given by (3) and the number of permutations $b^{(p)}(S)$ with descent set $S$ given by (4). The descent set of a permutation $\pi \in S_{p}$ is defined as $D(\pi)=\{i: \pi(i)>\pi(i+1), 1 \leq$ $i \leq p-1\}$. It is known, cf. [3, p. 69], that the number of permutations $\pi \in S_{p}$ with a given descent set $S=\left(s_{1}, \ldots, s_{m}\right), 1 \leq s_{1}<\cdots<s_{m} \leq p-1$, can be obtained by the following inclusion-exclusion formula:

$$
\begin{aligned}
b^{(p)}(S) & =b^{(p)}\left(s_{1}, \ldots, s_{m}\right) \\
& =\sum_{1 \leq i_{1}<\cdots<i_{j} \leq m}(-1)^{m-j}\left(\begin{array}{c}
p \\
s_{i_{1}}, s_{i_{2}}-s_{i_{1}}, \ldots, s_{i_{j}}-s_{i_{j-1}}, p-s_{i_{j}}
\end{array}\right)
\end{aligned}
$$

Theorem 1. The coefficient $a_{j_{1} \ldots j_{p-1}}^{(p)}$ given by (2) and (3) is equal to the number of permutations $b^{(p)}(S)$ with descent set $S$ given by (4).

Proof. It is enough to show that the numbers $b^{(p)}(S)$ satisfy the same recursion (5) as the numbers $a_{j_{1} \ldots j_{p-1}}^{(p)}$ do, that is,

$$
b^{(p)}\left(s_{1}, \ldots, s_{m}\right)=\sum_{l=0}^{m}\left(\begin{array}{l}
p \\
s_{l}
\end{array}\right)(-1)^{m-l} b^{\left(s_{l}\right)}\left(s_{1}, \ldots, s_{l-1}\right),
$$

where, by definition, $s_{0}=0$ and $b^{\left(s_{0}\right)}=b^{(0)}=1$.

To show this, let us substitute the inclusion-exclusion formula (6) into the right hand side of (7):

$$
\begin{aligned}
& \sum_{l=0}^{m}\left(\begin{array}{c}
p \\
s_{l}
\end{array}\right)(-1)^{m-l} b^{\left(s_{l}\right)}\left(s_{1}, \ldots, s_{l-1}\right) \\
& =(-1)^{m}+\sum_{l=1}^{m}\left(\begin{array}{c}
p \\
s_{l}
\end{array}\right)(-1)^{m-l} \sum_{1 \leq i_{1}<\cdots<i_{j} \leq l-1}(-1)^{l-1-j}\left(\begin{array}{c}
s_{l} \\
s_{i_{1}}, s_{i_{2}}-s_{i_{1}}, \ldots, s_{l}-s_{i_{j}}
\end{array}\right) \\
& =(-1)^{m}+\sum_{l=1}^{m} \sum_{1 \leq i_{1}<\cdots<i_{j}<l}^{p}(-1)^{m-j-1}\left(\begin{array}{c}
s_{i_{1}}, s_{i_{2}}-s_{i_{1}}, \ldots, s_{l}-s_{i_{j}}, p-s_{l}
\end{array}\right) \\
& =(-1)^{m}+\sum_{1 \leq i_{1}<\cdots<i_{j}<l \leq m}(-1)^{m-(j+1)}\left(\begin{array}{c}
p \\
s_{i_{1}}, s_{i_{2}}-s_{i_{1}}, \ldots, s_{l}-s_{i_{j}}, p-s_{l}
\end{array}\right) \\
& =\sum_{\left(\leq i_{1}<\cdots<i_{r} \leq m\right.} p_{(-1)^{m-r}}\left(\begin{array}{c}
p \\
s_{i_{1}}, s_{i_{2}}-s_{i_{1}}, \ldots, s_{i_{r}}-s_{i_{r-1}}, p-s_{i_{r}}
\end{array}\right)=b^{(p)}\left(s_{1}, \ldots, s_{m}\right) .
\end{aligned}
$$

This proves (7), and so completes the proof.

Lemma 1 described a recursion that uses all previous rows of Table 1 to compose the elements of a new row. In the next lemma we show a recursion that uses only the previous row and which is an analog of the recursion formula in the ordinary Pascal's triangle: $\left(\begin{array}{l}p \\ k\end{array}\right)=\left(\begin{array}{c}p-1 \\ k-1\end{array}\right)+\left(\begin{array}{c}p-1 \\ k\end{array}\right)$. Interestingly, an application of this identity is a key step in the following algebraic proof as well. We also give a simple combinatorial proof which basically translates the well-known method by which permutations in $S_{p}$ can be obtained from permutations in $S_{p-1}$ by adjoining the number $p$. 
Lemma 2. The following recursion holds for any $p \geq 2$ and multiindex $\left(j_{1}, \ldots, j_{p-1}\right) \in$ $\{0,1\}^{p-1}$ :

$$
a_{j_{1} \ldots j_{p-1}}^{(p)}=\sum_{i=1}^{p-1} \delta_{i} a_{j_{1}^{(i)} \ldots j_{p-2}^{(i)}}^{(p-1)}=\sum_{\left(i_{1}, \ldots, i_{p-2}\right) \in L\left(j_{1}, \ldots, j_{p-1}\right)} a_{i_{1} \ldots i_{p-2}}^{(p-1)}
$$

where $a^{(1)}=1, \delta_{i}=\left|j_{i}-j_{i-1}\right|$ for $i \geq 2, \delta_{1}=1, j_{k}^{(i)}=j_{k}$ for $1 \leq k \leq i-1$, $j_{k}^{(i)}=j_{k+1}$ for $i \leq k \leq p-2$, and $L\left(j_{1}, \ldots, j_{p-1}\right)$ is the set of all distinct binary sequences obtained from $\left(j_{1}, \ldots, j_{p-1}\right)$ by deleting exactly one digit. For example, $a_{0110}^{(5)}=11=a_{110}^{(4)}+a_{010}^{(4)}+a_{011}^{(4)}$.

Proof. First we prove the second equality in (8). For this it is enough to show that if the same binary sequence is obtained from $\left(j_{1}, \ldots, j_{p-1}\right)$ when eliminating either the $k$ th or the $l$ th digit $(k<l)$, then all digits between the $k$ th and $l$ th (including these two) are uniformly either 0's or 1's (a run of 0's or 1's). Therefore, the two recursions given in (8) are the same.

Consider a multiindex $\left(j_{1}, \ldots, j_{k-1}, j_{k}, \ldots, j_{l}, j_{l+1}, \ldots, j_{p-1}\right) \in\{0,1\}^{p-1}$. Suppose that we get the same binary sequence by deleting $j_{k}$ and $j_{l}$, respectively: $\left(j_{1}, \ldots, j_{k-1}, j_{k+1}, \ldots, j_{l}, j_{l+1}, \ldots, j_{p-1}\right)=\left(j_{1}, \ldots, j_{k-1}, j_{k}, \ldots, j_{l-1}, j_{l+1}, \ldots, j_{p-1}\right)$. Then $j_{k}=j_{k+1}=\cdots=j_{l-1}=j_{l}$, so the second equality in (5) really holds.

Now it remains to show the first equality in (8), that is, the recursion itself.

A combinatorial proof of the recursion. Given a binary sequence $\left(j_{1}, \ldots, j_{p-1}\right)$, let us remove a single 1 from a run of 1's or a single 0 from a run of 0's. Count the number of permutations in $S_{p-1}$ determined by the resulting multiindex $\left(i_{1}, \ldots, i_{p-2}\right)$. This number is $a_{i_{1} \ldots i_{p-2}}^{(p-1)}$ by Theorem 1. We want to show that there is a uniquely determined adjoining of the number $p$ to any such permutation from $S_{p-1}$ to obtain a permutation from $S_{p}$ corresponding to the original multindex $\left(j_{1}, \ldots, j_{p-1}\right)$.

If a 0 was deleted from a run of 0 's, the number $p$ should be inserted right after the number at the position of the first 1 following the affected run of 0 's. (If the given run happens to be the last, $p$ is inserted as the last number.) When a 1 was deleted from a run of 1's, the number $p$ should be inserted right after the number at the position of the last 0 preceding the affected run of 1's. (If the given run happens to be the first, $p$ is inserted as the first number.) Since these insertions are the only ones that reconstruct the original descent set, the recursion is proved.

An algebraic proof of the recursion. We are going to proceed by induction over $p$. Thus suppose that the recursion holds for all multiindices of lengths smaller than $p-1$. First we use the recursion of Lemma 1 for the terms in the right side of (8), then we change the order of summation to obtain

$$
\begin{aligned}
\sum_{i=1}^{p-1} \delta_{i} a_{j_{1}^{(i)} \ldots j_{p-2}^{(i)}}^{(p-1)}= & \sum_{i=1}^{p-1} \delta_{i} \sum_{k=0}^{p-2}\left(\begin{array}{c}
p-1 \\
k
\end{array}\right) j_{k}^{(i)}(-1)^{j_{k+1}^{(i)}+\cdots+j_{p-2}^{(i)}} a_{j_{1}^{(i)} \ldots j_{k-1}^{(i)}}^{(k)} \\
= & \sum_{k=0}^{p-2}\left(\begin{array}{c}
p-1 \\
k
\end{array}\right)\left\{j_{k+1}(-1)^{j_{k+2}+\cdots+j_{p-1}} \sum_{i=1}^{k} \delta_{i} a_{j_{1}^{(i)} \ldots j_{k-1}^{(i)}}^{(k)}\right. \\
& \left.+j_{k}(-1)^{j_{k+1}+\cdots+j_{p-1}} a_{j_{1} \ldots j_{k-1}}^{(k)} \sum_{i=k+1}^{p-1} \delta_{i}(-1)^{j_{i}}\right\}
\end{aligned}
$$


Here in the last expression, one may use the induction hypothesis for the first sum. In the second sum observe that $\delta_{i}(-1)^{j_{i}}$ is 1 if $j_{i-1}=1$ and $j_{i}=0$, it is -1 if $j_{i-1}=0$ and $j_{i}=1$, and it equals 0 otherwise. Hence we get the identity $j_{k} \sum_{i=k+1}^{p-1} \delta_{i}(-1)^{j_{i}}=j_{k}\left(1-j_{p-1}\right)$. Thus one obtains that

$$
\begin{aligned}
& \sum_{i=1}^{p-1} \delta_{i} a_{j_{1}^{(i)} \ldots j_{p-2}^{(i)}}^{(p-1)}=\sum_{k=0}^{p-2}\left\{\left(\begin{array}{c}
p-1 \\
k
\end{array}\right) j_{k+1}(-1)^{j_{k+2}+\cdots+j_{p-1}} a_{j_{1} \ldots j_{k}}^{(k+1)}\right. \\
& \left.+\left(\begin{array}{c}
p-1 \\
k
\end{array}\right)\left(1-j_{p-1}\right) j_{k}(-1)^{j_{k+1}+\cdots+j_{p-1}} a_{j_{1} \ldots j_{k-1}}^{(k)}\right\} \\
& =\sum_{k=1}^{p-2}\left\{\left(\begin{array}{c}
p-1 \\
k-1
\end{array}\right)+\left(\begin{array}{c}
p-1 \\
k
\end{array}\right)\left(1-j_{p-1}\right)\right\} j_{k}(-1)^{j_{k+1}+\cdots+j_{p-1}} a_{j_{1} \ldots j_{k-1}}^{(k)} \\
& +\left(1-j_{p-1}\right)(-1)^{j_{1}+\cdots+j_{p-1}}+\left(\begin{array}{c}
p-1 \\
p-2
\end{array}\right) j_{p-1} a_{j_{1} \ldots j_{p-2}}^{(p-1)} .
\end{aligned}
$$

To rewrite the terms above we use recursion (5) in the following case:

$$
a_{j_{1} \ldots j_{p-2}}^{(p-1)}=\sum_{k=0}^{p-2}\left(\begin{array}{c}
p-1 \\
k
\end{array}\right) j_{k}(-1)^{j_{k+1}+\cdots+j_{p-2}} a_{j_{1} \ldots j_{k-1}}^{(k)},
$$

plus the identity $-j_{p-1}(-1)^{j_{p-1}}=j_{p-1}$, and the recursion for binomial coefficients:

$$
\begin{aligned}
\sum_{i=1}^{p-1} \delta_{i} a_{j_{1}^{(i)} \ldots j_{p-2}^{(i)}}^{(p-1)}= & \sum_{k=1}^{p-2}\left\{\left(\begin{array}{l}
p-1 \\
k-1
\end{array}\right)+\left(\begin{array}{c}
p-1 \\
k
\end{array}\right)\right\} j_{k}(-1)^{j_{k+1}+\cdots+j_{p-1}} a_{j_{1} \ldots j_{k-1}}^{(k)} \\
& -j_{p-1}(-1)^{j_{p-1}}\left(a_{j_{1} \ldots j_{p-2}}^{(p-1)}-(-1)^{j_{1}+\cdots+j_{p-2}}\right) \\
& +\left(1-j_{p-1}\right)(-1)^{j_{1}+\cdots+j_{p-1}}+\left(\begin{array}{l}
p-1 \\
p-2
\end{array}\right) j_{p-1} a_{j_{1} \ldots j_{p-2}}^{(p-1)} \\
= & \sum_{k=0}^{p-1}\left(\begin{array}{l}
p \\
k
\end{array}\right) j_{k}(-1)^{j_{k+1}+\cdots+j_{p-1}} a_{j_{1} \ldots j_{k-1}}^{(k)}=a_{j_{1} \ldots j_{p-1}}^{(p)} .
\end{aligned}
$$

This completes the proof.

The results above imply that Table 1 has properties analogous to the ones of Pascal's triangle: each entry $a_{j_{1} \ldots j_{p-1}}^{(p)}$ is a positive integer, the first and the last entries, $a_{0 \ldots 0}^{(p)}$ and $a_{1 \ldots 1}^{(p)}$ are 1 , the table has symmetries $a_{j_{1} \ldots j_{p-1}}^{(p)}=a_{j_{p-1} \ldots j_{1}}^{(p)}$ and $a_{j_{1} \ldots j_{p-1}}^{(p)}=a_{1-j_{1} \ldots 1-j_{p-1}}^{(p)}$, and the sum of the $2^{p-1}$ entries in the $p$ th row is $p !$.

\section{Some remarks}

We mention that beside the indirect recursion (2), (3), direct recursions (5) and (8), and sieve formula (6), there are other methods as well in the literature that can be used for evaluating the coefficients $a_{j_{1} \ldots j_{p-1}}^{(p)}$. Here we mention two 
of them. Zabrocki [5] uses the following rather fast and practical 'splitting' recursion:

$$
a_{j_{1} \ldots j_{p-1}}^{(p)}=\sum_{\left\{k: j_{k-1}=0, j_{k}=1,1 \leq k \leq p\right\}}\left(\begin{array}{l}
p-1 \\
k-1
\end{array}\right) a_{j_{1} \ldots j_{k-2}}^{(k-1)} a_{j_{k+1} \ldots j_{p-1}}^{(p-k)},
$$

where, by definition, $j_{0}=0$ and $j_{p}=1$. Its verification is simple: this recursion divides the set of permutations in $S_{p}$ with descent set defined by the 1's in $\left(j_{1}, \ldots, j_{p-1}\right)$ into disjoint subsets, where the largest number $p$ is at the different local maxima $k$ where $j_{k-1}=0$ and $j_{k}=1$. Then there are $\left(\begin{array}{l}p-1 \\ k-1\end{array}\right)$ ways to pick the numbers to precede the largest, with $a_{j_{1} \ldots j_{k-2}}^{(k-1)}$ permutations, and $a_{j_{k+1} \ldots j_{p-1}}^{(p-k)}$ permutations for the numbers succeeding the largest, fitting the given descent set.

Another method (of mostly theoretical interest) can be based on MacMahon's determinant, cf. [3, p. 69]:

$$
a_{j_{1} \ldots j_{p-1}}^{(p)}=b^{(p)}\left(s_{1}, \ldots, s_{m}\right)=p ! \operatorname{det}\left[1 /\left(s_{j+1}-s_{i}\right) !\right], \quad(i, j) \in[0, m] \times[0, m] .
$$

where the descent set $S=\left(s_{1}, \ldots, s_{m}\right)$ is defined by (44), $s_{0}=0$, and $s_{m+1}=p$. This gives the recursion

$$
b^{(p)}\left(s_{1}, \ldots, s_{m}\right)=\frac{1}{s_{1} !} b^{(p)}\left(s_{2}-s_{1}, \ldots, s_{m}-s_{1}\right)-\frac{1}{s_{2} !} b^{(p)}\left(s_{3}-s_{2}, \ldots, s_{m}-s_{2}\right) .
$$

Obviously, the coefficients $a_{j_{1} \ldots j_{p-1}}^{(p)}$ are closely related to other important classifications of permutations as well. The Eulerian number $A(p, k)$ which counts the permutations in $S_{p}$ having exactly $k-1$ descents (that is, exactly $k$ runs), where $p \geq 1,1 \leq k \leq p$, can be written as

$$
A(p, k)=\sum_{\left\{\left(j_{1}, \ldots, j_{p-1}\right) \in\{0,1\}^{p-1}: \sum_{i=1}^{p-1} j_{i}=k-1\right\}} a_{j_{1} \ldots j_{p-1}}^{(p)} .
$$

Also, let $I(p, k)$ be the number of permutations in $S_{p}$ with $k$ inversions $\left(p \geq 1,0 \leq k \leq\left(\begin{array}{l}p \\ 2\end{array}\right)\right)$. By MacMahon's theorem, cf. [1, Section 5.1.1], $I(p, k)$ is equal to the number of permutations in $S_{p}$ with major index $k$, thus

$$
I(p, k)=\sum_{\left\{\left(j_{1}, \ldots, j_{p-1}\right) \in\{0,1\}^{p-1}: \sum_{i=1}^{p-1} i j_{i}=k\right\}} a_{j_{1} \ldots j_{p-1}}^{(p)} .
$$

Finally, let us express the generating function of the coefficients $a_{j_{1} \ldots j_{p-1}}^{(p)}$ by the help of a suitable recursive sequence $\left(e_{p}\right)_{p=1}^{\infty}$ given by (2). First, one can assign a positive integer $n$ to any $p \geq 1$ and multiindex $\left(j_{1}, \ldots, j_{p-1}\right)$ by the equation $n=2^{p-1}+\sum_{k=1}^{p-1} j_{k} 2^{k-1}$, in a one-to-one way. Then let us introduce the notation $\alpha_{n}=a_{j_{1} \ldots j_{p-1}}^{(p)}, n \geq 1$. This way the coefficients are arranged in a single sequence.

Second, take the variables (moments) $\mu_{k}=x^{2^{k-1}}$ for $k \geq 1, x \neq 1$, and let $\mu_{0}=1$. Then define the sequence $\left(e_{p}(x)\right)_{p=1}^{\infty}$ by (2):

$$
e_{p}(x)=\frac{1}{1-x^{2^{p-1}}}\left\{1+\sum_{k=1}^{p-1}\left(\begin{array}{l}
p \\
k
\end{array}\right) x^{2^{k-1}} e_{k}(x)\right\} .
$$


Also, using (3) and the definition of $n$ one obtains that

$$
x^{2^{p-1}} e_{p}(x)=\frac{1}{(1-x)\left(1-x^{2}\right)\left(1-x^{4}\right) \cdots\left(1-x^{2^{p-1}}\right)} \sum_{n=2^{p-1}}^{2^{p}-1} \alpha_{n} x^{n} .
$$

Hence the generating function of the sequence $\left(\alpha_{n}\right)_{n=1}^{\infty}$ can be expressed as

$$
\sum_{n=1}^{\infty} \alpha_{n} x^{n}=\sum_{p=1}^{\infty} x^{2^{p-1}}(1-x)\left(1-x^{2}\right)\left(1-x^{4}\right) \cdots\left(1-x^{2^{p-1}}\right) e_{p}(x) .
$$

\section{References}

[1] Knuth, D.E. (1973) The Art of Computer Programming. Vol. 3. Sorting and searching. Addison-Wesley, Reading, Mass.

[2] MacMahon, P.A. (1915) Combinatory Analysis. Vol. 1. Cambridge Univ. Press, Cambridge, England.

[3] Stanley, R. (1986) Enumerative Combinatorics. Vol. 1. Wadsworth and Brooks/Cole Mathematics Series, Monterey, Calif.

[4] Szabados, T. and Székely, B. (2003) An exponential functional of random walks. J. Appl. Prob. to appear.

[5] Zabrocki, M. (2001) Integer sequence A060351. On-line encyclopedia of integer sequences. http://www.research.att.com/ njas/sequences/Seis.html 